\title{
Control algorithms along relative equilibria of underactuated Lagrangian systems on Lie groups
}

Nordkvist, Nikolaj; Bullo, Francesco

Published in:

46th IEEE Conference on Decision and Control, 2007

Link to article, DOI:

10.1109/CDC.2007.4434093

Publication date:

2007

Document Version

Publisher's PDF, also known as Version of record

Link back to DTU Orbit

Citation (APA):

Nordkvist, N., \& Bullo, F. (2007). Control algorithms along relative equilibria of underactuated Lagrangian systems on Lie groups. In 46th IEEE Conference on Decision and Control, 2007 (pp. 6232-6237). IEEE. https://doi.org/10.1109/CDC.2007.4434093

\section{General rights}

Copyright and moral rights for the publications made accessible in the public portal are retained by the authors and/or other copyright owners and it is a condition of accessing publications that users recognise and abide by the legal requirements associated with these rights.

- Users may download and print one copy of any publication from the public portal for the purpose of private study or research.

- You may not further distribute the material or use it for any profit-making activity or commercial gain

- You may freely distribute the URL identifying the publication in the public portal 


\title{
Control algorithms along relative equilibria of underactuated Lagrangian systems on Lie groups
}

\author{
Nikolaj Nordkvist and Francesco Bullo
}

\begin{abstract}
We present novel algorithms to control underactuated mechanical systems. For a class of invariant systems on Lie groups, we design iterative small-amplitude control forces to accelerate along, decelerate along, and stabilize relative equilibria. The technical approach is based upon a perturbation analysis and the design of inversion primitives and composition methods. We illustrate the algorithms on an underactuated planar rigid body and on a satellite with two thrusters.
\end{abstract}

\section{INTRODUCTION}

In this paper we study control of underactuated mechanical systems on Lie groups. We concentrate on the construction of small-amplitude control forces that, when used iteratively, result in a given change of the velocity in the direction of a relative equilibrium, while the configuration changes as if the system had moved along the relative equilibrium. Perturbation analysis and Lie group theory play a crucial role in the analysis. Example systems to which the theory applies are an underactuated planar rigid body and a satellite with two thrusters. The motivation for studying underactuated systems is twofold; it gives rise to other design possibilities than a fully actuated system and it is appropriate in the situation of an actuator failure, meaning that such an analysis improves robustness to actuator failures.

A vast literature is available on mechanical control systems. Extensive research has focused on underactuated mechanical systems, especially in the context of controlled Lagrangians and Hamiltonians, e.g., see [2], [3] and subsequent works. Somehow less research is available for controlling systems along relative equilibria; a related spin-up problem is considered in [4], the theory of kinematic reductions is exposed in [5]. Since this document builds directly upon the work in [6] we refer the reader to that document for a literature survey relevant for control algorithms for underactuated Lagrangian systems on Lie groups. A generalization of the theory in [6] to a larger class of mechanical systems can be found in [7].

As main contribution of this paper, we propose algorithms to compute small amplitude control forces that speed up, slow down, or stabilize, an underactuated system along a relative equilibrium. This task is not accounted for in the

A revised version of this document has been submitted as a technical note to the IEEE Transactions on Automatic Control [1].

Nikolaj Nordkvist is with the Department of Mathematics, Technical University of Denmark, Matematiktorvet, Building 303 S, DK-2800 Kgs. Lyngby, Denmark n.nordkvistemat.dtu.dk

Francesco Bullo is with the Mechanical Engineering Department and with the Center for Control, Dynamical Systems and Computation at the University of California at Santa Barbara, 2338 Engineering Bldg II, Santa Barbara, CA 93106-5070, USA bullo@engineering.ucsb. edu framework developed in [6] which focused on velocities close to zero. The main advantage of the proposed approach is its applicability to systems that are not linearly controllable; the main limitation is that part of the results are applicable only to $n$-dimensional systems with $(n-1)$ controls.

This paper is organized as follows. First, in Section II, we review the mathematical model of simple mechanical control systems on Lie groups, as described in [5], and perform perturbation analysis for small amplitude forcing and initial velocity close to a relative equilibrium. Based on this analysis Section III presents the design of two local inversion maps. Section IV presents methods to compose the inversion maps into a motion primitive and give the construction of control algorithms based on this motion primitive. In Section V we illustrate the approach by applying the algorithms numerically to an underactuated planar rigid body and the satellite with two thrusters, and we end the note by summarizing the results in a conclusion.

\section{MATHEMATICAL MODEL AND PERTURBATION ANALYSIS NEAR A RELATIVE EQUILIBRIUM}

A simple mechanical control system on a Lie group is a mechanical system which has as configuration manifold an $n$ dimensional Lie group $G$, with Lie algebra $\mathfrak{g}$, and Lagrangian equal to the kinetic energy which is defined by an inertia tensor $\mathbb{I}: \mathfrak{g} \rightarrow \mathfrak{g}^{*}$. We assume that $G$ is a matrix Lie group with identity element id and adjoint map $\operatorname{Ad}_{g}: \mathfrak{g} \rightarrow \mathfrak{g}$ associated to each $g \in G$. Such a system has dynamics given by

$$
\begin{aligned}
\dot{g} & =g \cdot \xi \\
\mathbb{I} \dot{\xi} & =\operatorname{ad}_{\xi}^{*} \mathbb{I} \xi+\sum_{i=1}^{m} f_{i} u_{i}(t),
\end{aligned}
$$

where $g \in G$ is the configuration, $\xi \in \mathfrak{g}$ is the bodyfixed velocity, $\operatorname{ad}_{\xi}: \mathfrak{g} \rightarrow \mathfrak{g}$ is the adjoint operator and $\operatorname{ad}_{\xi}^{*}: \mathfrak{g}^{*} \rightarrow \mathfrak{g}^{*}$ its dual, $f_{i} \in \mathfrak{g}^{*}$ defines the $i$ th bodyfixed force, and $u: \mathbb{R} \rightarrow \mathbb{R}^{m}$ is bounded and measurable and gives the resultant force on the system according to $\sum_{i=1}^{m} f_{i} u_{i}(t)$. In what follows, $\Sigma=\left(G, \mathbb{I},\left\{f_{1}, \ldots, f_{m}\right\}\right)$ denotes this mechanical control system.

We define the symmetric product $\langle\cdot: \cdot\rangle: \mathfrak{g} \times \mathfrak{g} \rightarrow \mathfrak{g}$ by

$$
\langle\xi: \eta\rangle:=-\mathbb{I}^{-1}\left(\operatorname{ad}_{\xi}^{*} \mathbb{I} \eta+\operatorname{ad}_{\eta}^{*} \mathbb{I} \xi\right) .
$$

Defining $b_{i}:=\mathbb{I}^{-1} f_{i}, i \in\{1, \ldots, m\}$, the dynamic equa- 
tion (2) can be written as

$$
\dot{\xi}=-\frac{1}{2}\langle\xi: \xi\rangle+\sum_{i=1}^{m} b_{i} u_{i}(t)
$$

Remark 1 (Simplifying convention): It is well known that $\mathfrak{g}$ is an $n$-dimensional vector space. In what follows, we make no distinction between $\mathfrak{g}$ and $\mathbb{R}^{n}$. This we do in order to express a vector in $\mathfrak{g}$ as a column vector in $\mathbb{R}^{n}$ and represent a linear map on $\mathfrak{g}$ as a matrix. This choice is not to be confused with an irrelevance of the Lie algebra structure since this is far from being the case.

A relative equilibrium for $\Sigma$ is a curve $t \mapsto g_{0} \exp \left(t \xi_{\text {re }}\right) \in$ $G$, for $g_{0} \in G$ and $\xi_{\text {re }} \in \mathbb{R}^{n}$, that is a solution to the dynamics (1), (2) for zero input $u$. It is easy to see that $t \mapsto g_{0} \exp \left(t \xi_{\text {re }}\right)$ is a relative equilibrium if and only if $\left\langle\xi_{\text {re }}: \xi_{\text {re }}\right\rangle=0$. It is convenient to call relative equilibrium both the curve $t \mapsto g_{0} \exp \left(t \xi_{\text {re }}\right)$ and the vector $\xi_{\text {re }}$. Given a relative equilibrium $\xi_{\text {re }}$, we define the linear map $A_{\text {re }}: \mathbb{R}^{n} \rightarrow$ $\mathbb{R}^{n}$ by $A_{\text {re }} \eta:=-\left\langle\xi_{\text {re }}: \eta\right\rangle$.

We are interested in control signals $u \in C^{0}\left([0,2 \pi], \mathbb{R}^{m}\right)$ of the form

$$
u(t)=\epsilon u^{1}(t)+\epsilon^{2} u^{2}(t), \quad 0<\epsilon \ll 1,
$$

where $u^{i} \in C^{0}\left([0,2 \pi], \mathbb{R}^{m}\right)$. Accordingly, we define $b^{j}(t):=\sum_{i=1}^{m} b_{i} u_{i}^{j}(t), j \in\{1,2\}$. In the perturbation analysis it will be convenient to define, for $f \in C^{0}\left([0,2 \pi], \mathbb{R}^{n}\right)$ and $\sigma \in \mathbb{R}$

$$
\bar{f}^{\sigma}(t):=\int_{0}^{t} e^{\sigma A_{\mathrm{re}}(t-s)} f(s) \mathrm{d} s, \quad \bar{f}(t):=\bar{f}^{0}(t) .
$$

In what follows, $s$ and $\tau$ will be used as integration variables only.

Proposition 2 (Perturbation analysis): Let $\Sigma$ be a mechanical control system on a Lie group with a relative equilibrium $\xi_{\text {re }}$ and corresponding matrix $A_{\text {re }}$. For $0<\epsilon \ll 1$ and $\sigma>0$, let $[0,2 \pi] \ni t \mapsto(g(t), \xi(t))$ be the solution to (1) and (3) with $t \mapsto \sum_{i}^{m} b_{i} u_{i}(t)=\epsilon b^{1}(t)+\epsilon^{2} b^{2}(t)$ and from initial velocity $\xi(0)=\sigma \xi_{\text {re }}+\epsilon^{2} \xi_{0}^{2}$, for $\xi_{0}^{2}=\mathcal{O}(1)$, and initial configuration $g(0)=\mathrm{id}$. Let $h(t):=g(t) \cdot \exp \left(-t \sigma \xi_{\text {re }}\right)$ and let $x(t):=\log (h(t))$ be the exponential coordinates of $h$. Then, for $t \in[0,2 \pi]$, it holds that $\xi(t, \epsilon)=\xi^{0}(t)+\epsilon \xi^{1}(t)+$ $\epsilon^{2} \xi^{2}(t)+\mathcal{O}\left(\epsilon^{3}\right)$ with

$$
\begin{aligned}
& \xi^{0}(t)=\sigma \xi_{\text {re }}, \\
& \xi^{1}(t)={\overline{b^{1}}}^{\sigma}(t), \\
& \xi^{2}(t)=e^{\sigma A_{\text {re }} t} \xi_{0}^{2}-\frac{1}{2}{\left.\overline{\left\langle\bar{b}^{1}\right.}:{\overline{b^{1}}}^{\sigma}\right\rangle}^{\sigma}(t)+{\overline{b^{2}}}^{\sigma}(t),
\end{aligned}
$$

and $x(t, \epsilon)=\epsilon x^{1}(t)+\epsilon^{2} x^{2}(t)+\mathcal{O}\left(\epsilon^{3}\right)$ with

$$
\begin{aligned}
& x^{1}(t)=\overline{\operatorname{Ad}_{\exp \left(s \sigma \xi_{\text {re }}\right)}\left({\overline{b^{1}}}^{\sigma}(s)\right)(t)}, \\
& x^{2}(t)=\overline{\operatorname{Ad}_{\exp \left(s \sigma \xi_{\text {re }}\right.}\left(e^{\sigma A_{\text {re }} s} \xi_{0}^{2}\right)}(t)
\end{aligned}
$$

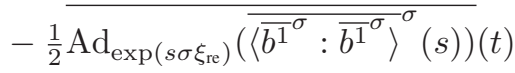

$$
\begin{aligned}
& +\overline{\operatorname{Ad}}_{\exp \left(s \sigma \xi_{\text {re }}\right)}\left({\overline{b^{2}}}^{\sigma}(s)\right)(t) \\
& -\frac{1}{2} \overline{\left[\operatorname{Ad}_{\exp \left(s \sigma \xi_{\text {re }}\right)}\left({\overline{b^{1}}}^{\sigma}(s)\right), \overline{\operatorname{Ad}_{\exp \left(\tau \sigma \xi_{\text {re }}\right)}\left({\overline{b^{1}}}^{\sigma}(\tau)\right)}(s)\right]}(t) .
\end{aligned}
$$

Proof: Since the input is analytic in $\epsilon$ so is the solution $\xi(t)=\sum_{j=0}^{+\infty} \epsilon^{j} \xi^{j}(t)$. Inserting the expansions for $\xi$ into equation (3) and collecting terms of same order we compute

$$
\begin{aligned}
& \dot{\xi}^{0}=-\frac{1}{2}\left\langle\xi^{0}: \xi^{0}\right\rangle, \quad \dot{\xi}^{1}=-\left\langle\xi^{0}: \xi^{1}\right\rangle+b^{1}(t), \\
& \dot{\xi}^{2}=-\left\langle\xi^{0}: \xi^{2}\right\rangle-\frac{1}{2}\left\langle\xi^{1}: \xi^{1}\right\rangle+b^{2}(t) .
\end{aligned}
$$

Inserting the initial condition then gives

$$
\begin{aligned}
\xi^{0}(t) & =\sigma \xi_{\mathrm{re}}, \quad \xi^{1}(t)={\overline{b^{1}}}^{\sigma}(t) \\
\xi^{2}(t) & =e^{\sigma A_{\mathrm{re}} t} \xi_{0}^{2}-\frac{1}{2} \overline{\left\langle\xi^{1}: \xi^{1}\right\rangle}(t)+{\overline{b^{2}}}^{\sigma}(t) \\
& =e^{\sigma A_{\mathrm{re}} t} \xi_{0}^{2}-\frac{1}{2}{\left.\overline{\left\langle\bar{b}^{1}\right.}: \bar{b}^{\sigma}\right\rangle}^{\sigma}(t)+{\overline{b^{2}}}^{\sigma}(t) .
\end{aligned}
$$

Since $g$ is a solution to the kinematic equation (1), it follows that

$$
\begin{aligned}
\dot{h} & =\dot{g} \cdot \exp \left(-t \sigma \xi_{\mathrm{re}}\right)-g \cdot \exp \left(-t \sigma \xi_{\mathrm{re}}\right) \cdot \sigma \xi_{\mathrm{re}} \\
& =g \cdot \xi \cdot \exp \left(-t \sigma \xi_{\mathrm{re}}\right)-h \cdot \sigma \xi_{\mathrm{re}} \\
& =h \cdot\left(\exp \left(t \sigma \xi_{\mathrm{re}}\right) \cdot \xi \cdot \exp \left(-t \sigma \xi_{\mathrm{re}}\right)-\sigma \xi_{\mathrm{re}}\right) \\
& =h \cdot\left(\operatorname{Ad}_{\exp \left(t \sigma \xi_{\mathrm{re}}\right)}(\xi)-\sigma \xi_{\mathrm{re}}\right) \\
& =h \cdot\left(\operatorname{Ad}_{\exp \left(t \sigma \xi_{\mathrm{re}}\right)}\left(\sigma \xi_{\mathrm{re}}+\epsilon \xi^{1}+\epsilon^{2} \xi^{2}+\mathcal{O}\left(\epsilon^{3}\right)\right)-\sigma \xi_{\mathrm{re}}\right) \\
& =h \cdot \operatorname{Ad}_{\exp \left(t \sigma \xi_{\mathrm{re}}\right)}\left(\epsilon \xi^{1}+\epsilon^{2} \xi^{2}+\mathcal{O}\left(\epsilon^{3}\right)\right) .
\end{aligned}
$$

If we define $\zeta(t):=\operatorname{Ad}_{\exp \left(t \sigma \xi_{\text {re }}\right)}\left(\epsilon \xi^{1}+\epsilon^{2} \xi^{2}+\mathcal{O}\left(\epsilon^{3}\right)\right)$, then we have, according to [8], that

$$
x(t)=\bar{\zeta}(t)-\frac{1}{2} \overline{[\zeta, \bar{\zeta}]}(t)+\mathcal{O}\left(\epsilon^{3}\right) .
$$

Using $x=\epsilon x^{1}+\epsilon^{2} x^{2}+\mathcal{O}\left(\epsilon^{3}\right)$ we achieve the result on $x^{1}$ and $x^{2}$ by inserting the expression for $\zeta$ into equation (4).

\section{DESIGN: LOCAL INVERSION PRIMITIVES}

For a mechanical control system $\Sigma=\left(G, \mathbb{I},\left\{f_{1}, \ldots, f_{m}\right\}\right)$ with relative equilibrium $\xi_{\text {re }}$ and corresponding matrix $A_{\text {re }}$, we present the following assumptions. First, we make the standing assumption that $\xi_{\text {re }} \notin \operatorname{span}\left\{b_{1}, \ldots, b_{m}\right\}$, otherwise the theory of kinematic reductions [5] is readily applicable and the control problems we consider below are trivial.

Assumption 1 (Lack of linear controllability): The subspace $\operatorname{span}\left\{b_{1}, \ldots, b_{m}\right\}$ is invariant under the linear map $A_{\text {re }}$, that is, $\left\langle\xi_{\text {re }}: b_{i}\right\rangle \in \operatorname{span}\left\{b_{1}, \ldots, b_{m}\right\}$, for $i \in\{1, \ldots, m\}$.

Assumption 2 (Nonlinear controllability): The subspace $\operatorname{span}\left\{b_{i},\left\langle b_{i}: b_{j}\right\rangle \mid i, j \in\{1, \ldots, m\}\right\}$ is full rank and $\left\langle b_{i}: b_{i}\right\rangle \in \operatorname{span}\left\{b_{1}, \ldots, b_{m}\right\}$, for $i \in\{1, \ldots, m\}$.

Assumption 3: $\left\langle\xi_{\mathrm{re}}:\left\langle b_{i}: b_{j}\right\rangle\right\rangle \in \operatorname{span}\left\{b_{1}, \ldots, b_{m}\right\}$, for $i, j \in\{1, \ldots, m\}$ with $i \neq j$.

Assumption 4: The subspace $\operatorname{span}\left\{b_{1}, \ldots, b_{m}\right\}$ is invariant under the linear map $\operatorname{ad}_{\xi_{\text {re }}}$.

Assumption 2 is the same controllability assumption adopted in [6]. If we define the matrix $B:=\left[\begin{array}{lll}b_{1} & \cdots & b_{m}\end{array}\right] \in \mathbb{R}^{n \times m}$, then Assumption 1 is equivalent to the existence of a matrix $Q \in \mathbb{R}^{m \times m}$ such that $A_{\mathrm{re}} B=B Q$, and in turn $e^{A_{\mathrm{re}}} B=$ $B e^{Q}$. Similarly, Assumption 4 is equivalent to the existence of a matrix $M \in \mathbb{R}^{m \times m}$ such that $\operatorname{ad}_{\xi_{\mathrm{re}}} B=B M$. 
Given $Q \in \mathbb{R}^{m \times m}$, define $F_{Q}: C^{0}\left([0,2 \pi], \mathbb{R}^{m}\right) \rightarrow\{f \in$ $\left.C^{1}\left([0,2 \pi], \mathbb{R}^{m}\right) \mid f(0)=0\right\}$ by

$$
F_{Q}[u](t):=\int_{0}^{t} e^{Q(t-s)} u(s) \mathrm{d} s .
$$

Lemma 3 (Transformation of controls): The map $F_{Q}$ is invertible and its inverse is given as follows: if $w=$ $F_{Q}[u]$, then $u(t)=-Q w(t)+\dot{w}(t)$. Additionally, as in Assumption 1 , let $A_{\mathrm{re}}, B$ and $Q$ satisfy $A_{\mathrm{re}} B=B Q$. If $u \in C^{0}\left([0,2 \pi], \mathbb{R}^{m}\right)$ and $w=F_{\sigma Q}[u], \sigma \in \mathbb{R}$, then

$$
\overline{B u}^{\sigma}(t)=B w(t) \text {. }
$$

Proof: One-to-one correspondence between $u$ and $w$ is readily checked. We compute $\overline{B u}^{\sigma}(t)=$ $\int_{0}^{t} e^{\sigma A_{\mathrm{re}}(t-s)} B u(s) \mathrm{d} s=B \int_{0}^{t} e^{\sigma Q(t-s)} u(s) \mathrm{d} s=B w(t)$.

Definition 4 (Convenient forcing frequencies): Take $r=$ $\left\lceil\frac{n}{m}\right\rceil$. For $(i, h) \in\{1, \ldots, m\} \times\{1, \ldots, r\}$, select numbers $\alpha_{i h}$ in the set $\left\{0, \ldots, r m+\frac{1}{2} m(m-1)\right\}$ as follows:

$1: \mathcal{V}:=\emptyset ; \mathcal{I}:=\left\{1, \ldots, r m+\frac{1}{2} m(m-1)\right\}$

2: for $h \in\{1, \ldots, r\}$ and for $i \in\{1, \ldots, m\}$ do

3: $\quad \omega:=\min (\mathcal{I}) ; v:=\int_{0}^{2 \pi} \operatorname{Ad}_{\exp \left(s \sigma \xi_{\mathrm{re}}\right)} b_{i} \sin (\omega s) \mathrm{d} s$

4: $\quad$ if $v \in \operatorname{span}(\mathcal{V})$ then $\alpha_{i h}:=0$ else $\alpha_{i h}:=\omega ; \mathcal{I}:=$ $\mathcal{I} \backslash\{\omega\} ; \mathcal{V}:=\mathcal{V} \cup\{v\}$ end if

\section{5: end for}

Define $\mathcal{A}_{\sigma, \alpha}$ to be the $n \times r m$ matrix with $j$ th row

$$
\left[\mathcal{A}_{\sigma, \alpha}\right]_{j}:=\int_{0}^{2 \pi} \operatorname{Ad}_{\exp \left(s \sigma \xi_{\mathrm{re}}\right)}\left(b_{k} \sin \left(\alpha_{k h} s\right)\right) \mathrm{d} s,
$$

where $j=k+(h-1) m, k \in\{1, \ldots, m\}, h \in\{1, \ldots, r\}$. Next, for $(i, j) \in\{1, \ldots, m\}^{2}$, select numbers $\beta_{i j}$ as follows: for $i<j$ take $\beta_{i j} \in\left\{1, \ldots, r m+\frac{1}{2} m(m-1)\right\} \backslash$ $\left\{\alpha_{k h}\right\}_{(k, h) \in\{1, \ldots, m\} \times\{1, \ldots, r\}}$ all having distinct values, for $i>j$ take $\beta_{i j}=\beta_{j i}$, and for $i=j$ take $\beta_{i j}=0$.

Remark 5: In other words, the numbers $\alpha_{i j}$ are selected sequentially in such a way as to maximize the rank of $\mathcal{A}_{\sigma, \alpha}$. Note that, for $i, j, k, l \in\{1, \ldots, m\}$ and $h \in\{1, \ldots, r\}$, we have: (i) all nonzero $\alpha_{i h}$ are distinct, (ii) all nonzero $\alpha_{i h}$ are distinct from all nonzero $\beta_{j k}$, and (iii) $\beta_{i j}=\beta_{k l}$ if and only if $(i, j)=(k, l)$ or $(i, j)=(l, k)$.

Remark 6: The computations required by Definition 4 include checking that a vector belongs to a subspace. In practical numerical implementations it is sufficient to verify this condition up to a specified tolerance. It is convenient to choose this tolerance comparable with the accuracy of the control algorithms.

For $Z \in \mathbb{R}^{m \times m}$ define $\lambda: \mathbb{R}^{m \times m} \rightarrow \mathbb{R}^{m \times m}$ by

$$
\lambda_{j k}(Z):=\left\{\begin{array}{lll}
\operatorname{sign}\left(Z_{j k}\right) \sqrt{\left|Z_{j k}\right|}, & j<k, \\
0 & , & j=k, \\
\frac{1}{\pi} \sqrt{\left|Z_{k j}\right|} & , & j>k .
\end{array}\right.
$$

We are now able to obtain the following result.

Proposition 7 (speed_inversion): Let $\Sigma$ be a mechanical control system on a Lie group with a relative equilibrium $\xi_{\text {re }}$ and corresponding matrix $A_{\text {re }}$ and satisfying Assumptions 1,2 and 3. Let $Q \in \mathbb{R}^{m \times m}$ satisfy $A_{\mathrm{re}} B=B Q$. Let $\eta \in \mathbb{R}^{n}, \sigma \in \mathbb{R}$, and compute $z \in \mathbb{R}^{m}$ and $Z \in \mathbb{R}^{m \times m}$ as the pseudoinverse solution to

$\eta=\sum_{i=1}^{m} z_{i} b_{i}-\sum_{j=1}^{m-1} \sum_{k=j+1}^{m} Z_{j k}\left\langle b_{j}: b_{k}\right\rangle, \quad Z_{j k}=0$ for $j \geq k$.

Given $r, \alpha, \mathcal{A}_{\sigma, \alpha}$, and $\beta$ as in Definition 4 , let

$$
y_{j}(t):=\sum_{k=1}^{m} \lambda_{j k}(Z) \sin \left(\beta_{j k} t\right), \quad j \in\{1, \ldots, m\},
$$

and let $\gamma=\left(\gamma_{11}, \ldots, \gamma_{m 1}, \ldots, \gamma_{1 r}, \ldots, \gamma_{m r}\right)^{T}$ be the unique solution to

$$
\begin{aligned}
\mathcal{A}_{\sigma, \alpha} \gamma & =-\overline{\operatorname{Ad}_{\exp \left(s \sigma \xi_{\mathrm{re}}\right.}(B y(s))}(2 \pi), \\
\gamma_{i h} & =0 \text { if } \alpha_{i h}=0 \text { for }(i, h) \in\{1, \ldots, m\} \times\{1, \ldots, r\} .
\end{aligned}
$$

Additionally, if we take

$$
\begin{aligned}
& w_{j}^{1}(t)=y_{j}(t)+\sum_{l=1}^{r} \gamma_{j l} \sin \left(\alpha_{j l} t\right), \quad j \in\{1, \ldots, m\}, \\
& u^{1}(t)=F_{\sigma Q}^{-1}\left[w^{1}\right](t), \quad u^{2}(t)=\frac{1}{2 \pi} e^{\sigma Q(t-2 \pi)}(\chi+z),
\end{aligned}
$$

where $\chi \in \mathbb{R}^{m}$ is the unique solution to

$$
\begin{aligned}
B \chi= & \sum_{j=1}^{m-1} \sum_{k=j+1}^{m} \int_{0}^{2 \pi}\left(e^{\sigma A_{\mathrm{re}}(2 \pi-s)}-I\right) w_{j}^{1}(s) w_{k}^{1}(s) \mathrm{d} s\left\langle b_{j}: b_{k}\right\rangle \\
& +\frac{1}{2} \sum_{i=1}^{m} \int_{0}^{2 \pi} e^{\sigma A_{\mathrm{re}}(2 \pi-s)}\left(w_{i}^{1}(s)\right)^{2} \mathrm{~d} s\left\langle b_{i}: b_{i}\right\rangle,
\end{aligned}
$$

then $b^{1}(t)=B u^{1}(t)$ and $b^{2}(t)=B u^{2}(t)$ satisfy

$$
\begin{aligned}
\left.-\frac{1}{2}{\overline{\bar{b}^{1}}}^{\sigma}:{\overline{b^{1}}}^{\sigma}\right\rangle^{\sigma}(2 \pi)+{\overline{b^{2}}}^{\sigma}(2 \pi) & =\eta, \\
\operatorname{Ad}_{\exp \left(s \sigma \xi_{\mathrm{re}}\right)}\left({\overline{b^{1}}}^{\sigma}(s)\right)(2 \pi) & =0 .
\end{aligned}
$$

We call this map speed_inversion $(\sigma, \eta)=\left(b^{1}(t), b^{2}(t)\right)$.

Proof: Existence and uniqueness of the solution to (6) is a consequence of Assumptions 3 and 2. Regarding existence and uniqueness of the solution to (5), Definition 4 ensures that

$$
\overline{\operatorname{Ad}_{\exp \left(s \sigma \xi_{\mathrm{re}}\right.}(B y(s))}(2 \pi) \in \operatorname{Image}\left(\mathcal{A}_{\sigma, \alpha}\right) .
$$

Since every nonzero column in $\mathcal{A}_{\sigma, \alpha}$ contributes to the rank of $\mathcal{A}_{\sigma, \alpha}$, the entries of $\gamma$ corresponding to these will be unique. The remaining $\gamma$-values are defined to be 0 .

Regarding the proof of equation (8), direct calculations show that

$$
\begin{aligned}
\overline{\operatorname{Ad}_{\exp \left(s \sigma \xi_{\mathrm{re}}\right)}\left(\overline{b^{1}}{ }^{\sigma}(s)\right)}(2 \pi) & =\overline{\operatorname{Ad}_{\exp \left(s \sigma \xi_{\mathrm{re}}\right)}\left(B w^{1}(s)\right)}(2 \pi) \\
& =\mathcal{A}_{\sigma, \alpha} \gamma+\overline{\operatorname{Ad}_{\exp \left(s \sigma \xi_{\mathrm{re}}\right)}(B y(s))}(2 \pi) \\
& =0 .
\end{aligned}
$$


Regarding the proof of equation (7), from Lemma 3 we compute

$$
\begin{aligned}
\left\langle\bar{b}^{\sigma}: \bar{b}^{\sigma}\right\rangle(t)= & \left\langle\sum_{j=1}^{m} w_{j}^{1}(t) b_{j}: \sum_{k=1}^{m} w_{k}^{1}(t) b_{k}\right\rangle \\
= & 2 \sum_{j=1}^{m-1} \sum_{k=j+1}^{m} w_{j}^{1}(t) w_{k}^{1}(t)\left\langle b_{j}: b_{k}\right\rangle \\
& +\sum_{i=1}^{m}\left(w_{i}^{1}(t)\right)^{2}\left\langle b_{i}: b_{i}\right\rangle .
\end{aligned}
$$

Since all nonzero $\alpha$-values are distinct and are distinct from the $\beta$-values we have for $j<k$

$$
\begin{aligned}
\int_{0}^{2 \pi} w_{j}^{1}(t) w_{k}^{1}(t) \mathrm{d} t & =\sum_{l, q=1}^{m} \lambda_{j l}(Z) \lambda_{k q}(Z) \int_{0}^{2 \pi} \sin \left(\beta_{j l} t\right) \sin \left(\beta_{k q} t\right) \mathrm{d} t \\
& =\sum_{l, q=1}^{m} \lambda_{j l}(Z) \lambda_{k q}(Z) \delta_{\beta_{k q}}^{\beta_{j l}} \pi \\
& =\lambda_{j k}(Z) \lambda_{k j}(Z) \pi=Z_{j k} .
\end{aligned}
$$

By straightforward calculations we then obtain

$$
\begin{aligned}
\left.-\frac{1}{2}{\overline{\left\langle\bar{b}^{1}\right.}}^{\sigma}:{\overline{b^{1}}}^{\sigma}\right\rangle & (2 \pi)+{\overline{b^{2}}}^{\sigma}(2 \pi) \\
= & -\frac{1}{2} \int_{0}^{2 \pi} e^{\sigma A_{\mathrm{re}}(2 \pi-s)}\left\langle{\overline{b^{1}}}^{\sigma}:{\overline{b^{1}}}^{\sigma}\right\rangle(s) \mathrm{d} s \\
& +B \int_{0}^{2 \pi} e^{\sigma Q(2 \pi-s)} u^{2}(s) \mathrm{d} s \\
= & -\sum_{j=1}^{m-1} \sum_{k=j+1}^{m}\left(\int_{0}^{2 \pi} w_{j}^{1}(s) w_{k}^{1}(s) \mathrm{d} s\left\langle b_{j}: b_{k}\right\rangle\right. \\
& \left.+\int_{0}^{2 \pi}\left(e^{\sigma A_{\mathrm{re}}(2 \pi-s)}-I\right) w_{j}^{1}(s) w_{k}^{1}(s) \mathrm{d} s\left\langle b_{j}: b_{k}\right\rangle\right) \\
& -\frac{1}{2} \sum_{j=1}^{m} \int_{0}^{2 \pi} e^{\sigma A_{\mathrm{re}}(2 \pi-s)}\left(w_{j}^{1}(s)\right)^{2} \mathrm{~d} s\left\langle b_{j}: b_{j}\right\rangle \\
& +\sum_{i=1}^{m}\left(\chi_{i}+z_{i}\right) b_{i} \\
= & -\sum_{j=1}^{m-1} \sum_{k=j+1}^{m} Z_{j k}\left\langle b_{j}: b_{k}\right\rangle+\sum_{i=1}^{m} z_{i} b_{i}=\eta .
\end{aligned}
$$

Proposition 8 (configuration_inversion): Let $\Sigma$ be a mechanical control system on a Lie group with a relative equilibrium $\xi_{\text {re }}$ and corresponding matrix $A_{\text {re }}$ and satisfying Assumptions 1 and 4 . Let $Q, M \in \mathbb{R}^{m \times m}$ satisfy $A_{\mathrm{re}} B=$ $B Q$ and $\operatorname{ad}_{\xi_{\mathrm{re}}} B=B M$. If $\mu \in \mathbb{R}^{m}, \sigma \in \mathbb{R}$ and

$$
\begin{aligned}
& u^{1}(t)=0, \\
& u^{2}(t)=F_{\sigma Q}^{-1}\left[w^{2}\right](t), \quad w^{2}(t)=\frac{1}{\pi} e^{-\sigma M t} \mu \sin ^{2}(t),
\end{aligned}
$$

then $b^{1}(t)=B u^{1}(t)$ and $b^{2}(t)=B u^{2}(t)$ satisfy

$$
\begin{aligned}
& \left.-\frac{1}{2}{\overline{\bar{b}^{1}}}^{\sigma}:{\overline{b^{1}}}^{\sigma}\right\rangle^{\sigma}(2 \pi)+{\overline{b^{2}}}^{\sigma}(2 \pi)=0, \\
& \overline{\operatorname{Ad}_{\exp \left(s \sigma \xi_{\mathrm{re}}\right)}\left({\overline{b^{2}}}^{\sigma}(s)\right)}(2 \pi)=B \mu .
\end{aligned}
$$

We denote this map configuration_inversion $(\sigma, \mu)=$ $\left(b^{1}(t), b^{2}(t)\right)=\left(0, b^{2}(t)\right)$.

Proof: For $b^{1}(t)=0$ we have, using Lemma 3 and $w^{2}(t)=\frac{1}{\pi} e^{-\sigma M t} \mu \sin ^{2}(t)$, that

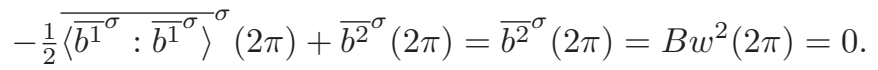

Using Assumption 4 and Lemma 3 we compute

$$
\begin{aligned}
\overline{\operatorname{Ad}_{\exp \left(s \sigma \xi_{\mathrm{re}}\right)}\left(\overline{b^{2}} \sigma(s)\right)}(2 \pi) & =\overline{\exp \left(s \sigma \operatorname{ad}_{\xi_{\mathrm{re}}}\right)\left(B w^{2}(s)\right)}(2 \pi) \\
& =\overline{B e^{\sigma M s} w^{2}(s)}(2 \pi) \\
& =\frac{1}{\pi} \overline{B \mu \sin ^{2}(s)}(2 \pi)=B \mu .
\end{aligned}
$$

\section{Design: GLOBAl MOTION ALGORITHMS}

The algorithm presented in this section requires the following additional assumption.

Assumption 5: The $n$ dimensional system $\Sigma$ has $n-1$ control forces, that is, $m=n-1$.

Remark 9: Assumption 5 together with the standing assumption $\xi_{\text {re }} \notin \operatorname{span}\left\{b_{1}, \ldots, b_{m}\right\}$ implies $\mathbb{R}^{n}=$ $\operatorname{span}\left\{b_{1}, \ldots, b_{m}, \xi_{\text {re }}\right\}$. Additionally, one can verify that Assumptions 5 and 1 together imply Assumption 3.

Define the projection operators $\mathcal{P}_{B}: \mathbb{R}^{n} \rightarrow \mathbb{R}^{n}$ and $\mathcal{P}_{\xi_{\text {re }}}:$ $\mathbb{R}^{n} \rightarrow \mathbb{R}^{n}$ by

$$
\mathcal{P}_{\xi_{\mathrm{re}}}(\nu):=\left(\nu \cdot \xi_{\mathrm{re}}\right) \xi_{\mathrm{re}}, \quad \mathcal{P}_{B}:=\mathrm{id}-\mathcal{P}_{\xi_{\mathrm{re}}} .
$$

where - is the dot product in $\mathbb{R}^{n}$ defined by requiring $\left\{b_{1}, \ldots, b_{m}, \xi_{\text {re }}\right\}$ to be an orthonormal basis. Notice that, under Assumption 4, these projection operators commute with $\operatorname{ad}_{\xi_{\mathrm{re}}}$. This allows us to construct the following motion primitive.

Proposition 10 (change_speed motion primitive): Let $\Sigma$ be a mechanical control system on a Lie group with a relative equilibrium $\xi_{\text {re }}$ and corresponding matrix $A_{\text {re }}$ and satisfying Assumptions 1, 2, 4, and 5. For $0<\epsilon \ll 1$, assume that

$$
\begin{aligned}
& g(0)=g_{0} \exp \left(\epsilon^{2} \nu_{\text {error }}\right), \\
& \xi(0)=\sigma \xi_{\text {re }}+\epsilon^{2} \xi_{\text {error }},
\end{aligned}
$$

for some $g_{0} \in G, \sigma \in \mathbb{R}, \nu_{\text {error }}, \xi_{\text {error }} \in \mathbb{R}^{n}$ with $\nu_{\text {error }}=\mathcal{O}(1)$ and $\xi_{\text {error }}=\mathcal{O}(1)$. If we take $\rho \in \mathbb{R}$,

$\left(b^{1}(t), b^{2}(t)\right)=$ $\left\{\begin{array}{ll}\text { speed_inversion }\left(\sigma, \rho \xi_{\text {re }}-e^{2 \pi \sigma A_{\text {re }}} \xi_{\text {error }}\right), & t \in[0,2 \pi] \\ \text { configuration_inversion }(\sigma, \mu) & , t \in[2 \pi, 4 \pi]\end{array}\right.$, and

$$
-B \mu=
$$$$
\operatorname{Ad}_{\exp \left(-2 \pi \sigma \xi_{\mathrm{re}}\right.} \mathcal{P}_{B}\left(\nu_{\text {error }}+\frac{1}{\epsilon^{2}} \log \left(g(0)^{-1} g(2 \pi) \exp \left(-2 \pi \sigma \xi_{\mathrm{re}}\right)\right)\right),
$$

then we obtain

$$
\begin{aligned}
& g(4 \pi)=g_{0}^{*} \exp \left(\epsilon^{2} \nu_{\text {error }}^{*},\right. \\
& \xi(4 \pi)=\left(\sigma+\epsilon^{2} \rho\right) \xi_{\text {re }}+\epsilon^{2} \xi_{\text {error }}^{*},
\end{aligned}
$$




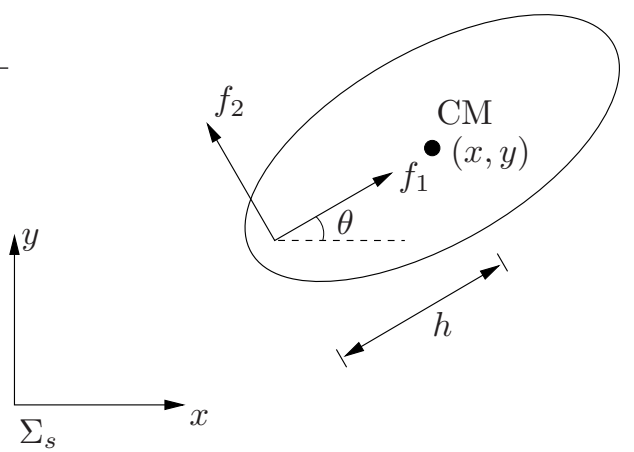

Fig. 1. The planar rigid body with two forces applied at a point a distance $h$ from the center of mass CM. $\Sigma_{s}$ denotes an inertial reference frame. $(\theta, x, y)$ are coordinates for the configuration of the body. The body reference frame (not depicted) is aligned with the direction of application of $f_{1}$ and $f_{2}$.

for some $\nu_{\text {error }}^{*}, \xi_{\text {error }}^{*} \in \mathbb{R}^{n}$ with $\mathcal{P}_{\xi_{\text {re }}}\left(\nu_{\text {error }}^{*}\right)=\mathcal{O}(1)$, $\mathcal{P}_{B}\left(\nu_{\text {error }}^{*}\right)=\mathcal{O}(\epsilon), \xi_{\text {error }}^{*}=\mathcal{O}(\epsilon)$ and for

$$
g_{0}^{*}=g_{0} \exp \left(\left(4 \pi \sigma+2 \pi \epsilon^{2} \rho\right) \xi_{\text {re }}+\epsilon^{2} \mathcal{P}_{\xi_{\text {re }}}\left(\nu_{\text {error }}\right)\right) .
$$

We denote this control map by $\left(\sigma+\epsilon^{2} \rho, g_{0}^{*}, \nu_{\text {error }}^{*}, \xi_{\text {error }}^{*}\right)=$ change_speed $\left(\epsilon, \sigma, \rho, g_{0}, \nu_{\text {error }}, \xi_{\text {error }}\right)$.

The proof of Proposition 10 is omitted but can be found in [9]. The proof uses Propositions 2, 7, 8 and the BakerCampbell-Hausdorff formula.

With this motion primitive we are able to construct the following algorithm that speeds up, slows down, or stabilizes, a system along a relative equilibrium.

Proposition 11 (speed_control algorithm): Let $\Sigma$ be a mechanical control system on a Lie group with a relative equilibrium $\xi_{\text {re }}$ and corresponding matrix $A_{\text {re }}$. Assume $\Sigma$ satisfies Assumptions 1, 2, 4, and 5 and take $0<\epsilon \ll 1$. Let $g(0), g_{0}, \nu_{\text {error }}, \sigma, \xi_{\text {error }}, \rho$ be as in Proposition 10 and let $N \in \mathbb{N}$.

Define the algorithm $\left(\sigma+\epsilon^{2} N \rho, g_{0}^{*}, \nu_{\text {error }}^{*}, \xi_{\text {error }}^{*}\right)=$ speed_control $\left(\epsilon, \sigma, \rho, N, g_{0}, \nu_{\text {error }}, \xi_{\text {error }}\right)$ by

$1: g_{0,1}:=g_{0} ; \nu_{\text {error }, 1}:=\nu_{\text {error }} ; \sigma_{1}:=\sigma ; \xi_{\text {error }, 1}:=\xi_{\text {error }}$;

2: for $k \in\{1, \ldots, N\}$ do

3: $\quad\left(\sigma_{k+1}, g_{0, k+1}, \nu_{\text {error }, k+1}, \xi_{\text {error }, k+1}\right):=$ change_speed $\left(\epsilon, \sigma_{k}, \rho, g_{0, k}, \nu_{\text {error }, k}, \xi_{\text {error }, k}\right)$

\section{4: end for}

5: $g_{0}^{*}=g_{0, N+1} ; \nu_{\text {error }}^{*}:=\nu_{\text {error }, N+1} ; \xi_{\text {error }}^{*}:=\xi_{\text {error }, N+1}$;

The final configuration and velocity after the execution of this algorithm are

$$
\begin{aligned}
& g(N 4 \pi)=g_{0}^{*} \exp \left(\epsilon^{2} \nu_{\text {error }}^{*}\right), \\
& \xi(N 4 \pi)=\left(\sigma+\epsilon^{2} N \rho\right) \xi_{\text {re }}+\epsilon^{2} \xi_{\text {error }}^{*},
\end{aligned}
$$

where $\nu_{\text {error }}^{*}, \xi_{\text {error }}^{*} \in \mathbb{R}^{n}, \mathcal{P}_{\xi_{\text {re }}}\left(\nu_{\text {error }}^{*}\right)=\mathcal{O}(1), \mathcal{P}_{B}\left(\nu_{\text {error }}^{*}\right)=$ $\mathcal{O}(\epsilon), \xi_{\text {error }}^{*}=\mathcal{O}(\epsilon)$, and

$g_{0}^{*}=g_{0} \exp \left(\left(\sigma T_{\text {final }}+\frac{1}{2} \rho \epsilon^{2} N T_{\text {final }}\right) \xi_{\text {re }}+\epsilon^{2} \sum_{k=1}^{N} \mathcal{P}_{\xi_{\text {re }}}\left(\nu_{\text {error }, k}\right)\right)$

The proof of this Proposition is omitted but can be found in [9]. It builds on Proposition 2 and 10.
Note that $\rho>0$ speeds up the system along the relative equilibrium, $\rho<0$ slows down the system, and $\rho=0$ stabilizes the system's motion along the relative equilibrium. We may select $N=\mathcal{O}\left(\frac{1}{\epsilon^{2}}\right)$ in Proposition 11 so that the absolute change in velocity along the relative equilibrium is of order $\mathcal{O}(1)$. Thus, it is possible to use the algorithm speed_control to change the velocity along the relative equilibrium from a given value to another independent of $\epsilon$.

\section{EXAMPLES}

The usefulness of the theory is illustrated in the following examples.

Example 12 (Planar rigid body): Consider a rigid body moving in the plane as described in [6]. The configuration manifold is $G=S E(2)$ with coordinates $(\theta, x, y)$. Let $m$ denote the mass of the body, $J$ its moment of inertia and $h$ the distance from the center of mass to the control forces. For $\left(\omega, v_{1}, v_{2}\right)^{T} \in \mathbb{R}^{3}$ we have that the adjoint operator is given by

$$
\operatorname{ad}_{\left(\omega, v_{1}, v_{2}\right)^{T}}=\left[\begin{array}{ccc}
0 & 0 & 0 \\
v_{2} & 0 & -\omega \\
-v_{1} & \omega & 0
\end{array}\right] .
$$

The inertia tensor has the representation $\mathbb{I}=\operatorname{diag}(J, m, m)$. With controls as in Figure 1 we have $b_{1}=\frac{1}{m} e_{2}$ and $b_{2}=$ $-\frac{h}{J} e_{1}+\frac{1}{m} e_{3}$, which gives $\left\langle b_{1}: b_{1}\right\rangle=0,\left\langle b_{2}: b_{2}\right\rangle=\frac{2 h}{J m} e_{2}$, and $\left\langle b_{1}: b_{2}\right\rangle=-\frac{h}{J m} e_{3}$. Assumption 2 is immediately seen to be satisfied. Choosing the relative equilibrium $\xi_{\text {re }}=e_{3}$ we have

$$
A_{\mathrm{re}}=\operatorname{ad}_{\xi_{\mathrm{re}}}=\left[\begin{array}{lll}
0 & 0 & 0 \\
1 & 0 & 0 \\
0 & 0 & 0
\end{array}\right],
$$

so Assumptions 3 and 4 are met. It is straightforward to calculate that $A_{\mathrm{re}} B=B Q$, with

$$
Q=-\frac{h m}{J}\left[\begin{array}{ll}
0 & 1 \\
0 & 0
\end{array}\right],
$$

so Assumption 1 is satisfied.

The $\gamma$-values can be calculated using Definition 4 to be $\alpha_{11}=\alpha_{12}=\alpha_{22}=0, \alpha_{21}=1, \gamma_{11}=\gamma_{12}=\gamma_{22}=$ 0 , and $\gamma_{21}=-\alpha_{21} \lambda_{21}(Z) / \beta$, where $\beta \in\{2,3,4,5\}$. Finally, the components of $\chi$ are found to be $\chi_{1}=$ $\pi h\left(\lambda_{21}(Z)^{2}+\gamma_{21}^{2}\right) / J$ and $\chi_{2}=0$.

Assumption 5 is immediately seen to be satisfied, so all the assumptions are met, and therefore we can apply the speed_control algorithm to speed up the system along $e_{3}$. The result of the speed_control algorithm applied to the planar rigid body can be seen in Figure 2 .

Example 13 (Satellite with two thrusters): Consider a satellite with two thrusters aligned with the first and second principal axes. The configuration manifold is $G=S O(3)$ and the equations of motion are of the form (1) and (3) where the symmetric product is given by $\langle\xi: \eta\rangle=\mathbb{I}^{-1}(\xi \times(\mathbb{I} \eta)+\eta \times(\mathbb{I} \xi))$, where $\mathbb{I}=\operatorname{diag}\left(J_{1}, J_{2}, J_{3}\right), J_{i}$ being the moment of inertia along the $i$ th principal axis, and $\times$ is the cross product. We have that $\left\langle e_{3}: e_{3}\right\rangle=0$, so $e_{3}$ is a relative equilibrium, and since 

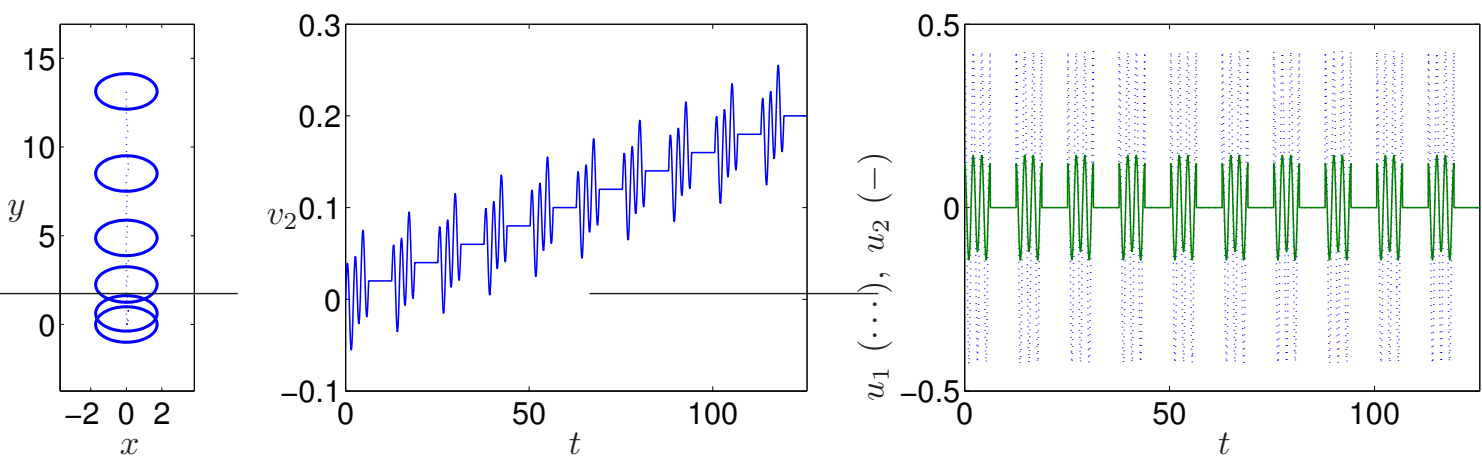

Fig. 2. speed_control applied to the planar rigid body with $\xi_{\text {re }}=e_{3}, \epsilon=0.1$, and $\rho=2$ and with initial conditions $(\theta, x, y)(0)=0, g_{0}=g(0)$, and $\left(\omega, v_{1}, v_{2}\right)(0)=0$. The dotted curve in the left figure corresponds to the motion of the center of mass and the ellipses corresponds to the planar body at time equidistant instances.

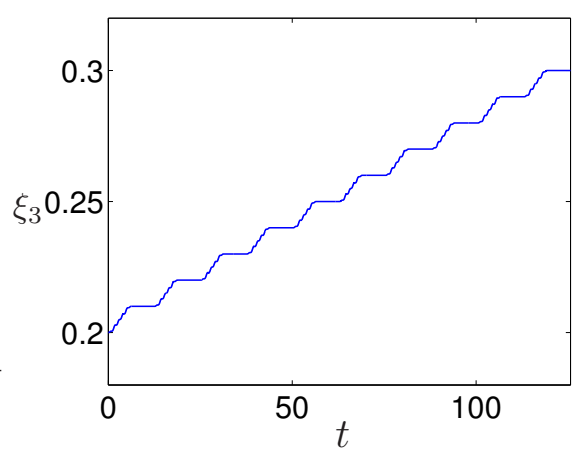

Fig. 3. speed_control applied to the satellite with two thrusters with $\xi_{\text {re }}=e_{3}, \epsilon=0.1$, and $\rho=1$ and with initial conditions $\xi(0)=(0,0,0.2)$ and $g_{0}=g(0)$.

$b_{1}=\frac{1}{J_{1}} e_{1}$ and $b_{2}=\frac{1}{J_{2}} e_{2}$ it is not possible to directly control the motion in the $e_{3}$ direction. With $\xi_{\text {re }}=e_{3}$ we compute

$$
A_{\mathrm{re}}=\left[\begin{array}{ccc}
0 & a_{12} & 0 \\
a_{21} & 0 & 0 \\
0 & 0 & 0
\end{array}\right],
$$

where $a_{12}=\frac{J_{2}-J_{3}}{J_{1}}$ and $a_{21}=\frac{J_{3}-J_{1}}{J_{2}}$. It is straightforward to calculate that $A_{\mathrm{re}} B=B Q$, with

$$
Q=\left[\begin{array}{cc}
0 & \frac{J_{2}-J_{3}}{J_{2}} \\
\frac{J_{3}-J_{1}}{J_{1}} & 0
\end{array}\right],
$$

so Assumption 1 is satisfied. From $\left\langle b_{1}: b_{1}\right\rangle=\left\langle b_{2}: b_{2}\right\rangle=$ 0 and $\left\langle b_{1}: b_{2}\right\rangle=\frac{J_{2}-J_{1}}{J_{1} J_{2} J_{3}} e_{3}$ we see that Assumption 2 is fulfilled if $J_{1} \neq J_{2}$. Assumption 3 is satisfied because $\left\langle e_{3}\right.$ : $\left.\left\langle b_{1}: b_{2}\right\rangle\right\rangle=\frac{J_{2}-J_{1}}{J_{1} J_{2} J_{3}}\left\langle e_{3}: e_{3}\right\rangle=0$. Since $\operatorname{ad}_{\xi} \eta=\xi \times \eta$ we see that also Assumption 4 is satisfied. Assumption 5 is immediately seen to be met. Thus, if $J_{1} \neq J_{2}$, the theory presented in this paper can be used to speed up the satellite with two thrusters along the unactuated principal axis $e_{3}$. This is illustrated in Figure 3.

\section{CONCLUSION}

In this note we have designed a motion control algorithm suitable for a class of invariant mechanical systems on Lie groups. Using small-amplitude control forces the algorithm solves the tasks of accelerating along, decelerating along, and stabilizing relative equilibria. The algorithm has been applied numerically to two example systems to illustrate the theory.

\section{Acknowledgements}

This material is based upon work supported in part by the NSF Award CMS-0442041. The authors thank Professor Poul G. Hjorth for his kind support.

\section{REFERENCES}

[1] N. Nordkvist and F. Bullo, "Control algorithms along relative equilibria of underactuated Lagrangian systems on Lie groups," IEEE Transactions on Automatic Control, Jan. 2007, submitted.

[2] A. M. Bloch, N. E. Leonard, and J. E. Marsden, "Controlled Lagrangians and the stabilization of mechanical systems. I. The first matching theorem," IEEE Transactions on Automatic Control, vol. 45 , no. 12, pp. 2253-2270, 2000.

[3] R. Ortega, A. J. van der Schaft, B. Maschke, and G. Escobar, "Interconnection and damping assignment passivity-based control of portcontrolled Hamiltonian systems," Automatica, vol. 38, no. 4, pp. 58596, 2002.

[4] D. Hristu, "The dynamics of a forced sphere-plate system," IEEE Transactions on Automatic Control, vol. 46, no. 5, pp. 678-86, 2001.

[5] F. Bullo and A. D. Lewis, Geometric Control of Mechanical Systems, ser. Texts in Applied Mathematics. New York: Springer Verlag, 2004 vol. 49.

[6] F. Bullo, N. E. Leonard, and A. D. Lewis, "Controllability and motion algorithms for underactuated Lagrangian systems on Lie groups," IEEE Transactions on Automatic Control, vol. 45, no. 8, pp. 1437-1454, 2000.

[7] S. Martínez and J. Cortés, "Motion control algorithms for simple mechanical systems with symmetry," Acta Applicandae Mathematicae, vol. 76, no. 3, pp. 221-264, 2003.

[8] A. T. Fomenko and R. V. Chakon, "Recursion relations for homogeneous terms of a convergent series of the logarithm of a multiplicative integral on Lie groups," Functional Analysis and its Applications, vol. 24, no. 1, pp. 48-58, 1990, translated from Russian.

[9] N. Nordkvist, "Motion control along relative equilibria," Ph.D. dissertation, Mathematics Department, Technical University of Denmark, Lyngby, Denmark, Sept. 2007. 